\title{
Erratum: Effective one-band approach for the spin splittings in quantum wells [Phys. Rev. B 95, 125303 (2017)]
}

\author{
P. S. Alekseev and M. O. Nestoklon $\odot$
}

(Received 16 November 2020; published 21 December 2020)

DOI: 10.1103/PhysRevB.102.239902

In the original paper, the numerical value of parameter $\zeta$ deduced from the calculated dependence of the two-dimensional electron spin splitting on the quantum well width was erroneously presented. This mistake originates from the incorrect conversion from the values used internally in our calculations. The correct value of parameter $\zeta$ obtained in the analysis is as follows: $\zeta=0.124 \mathrm{eV} \AA^{2}$. Also, the sign of parameter $\xi$ should be reversed: $\xi=1.5 \mathrm{eV} \AA^{3}$. The relative signs of these constants should be consistent with the fact that all three contributions $\beta_{b}, \beta_{\text {int }, 0}$, and $\beta_{\text {int }, 1}$ have the same sign. Note that all figures in the original paper are calculated using values $\zeta$ and $\xi$ presented here.

In Fig. 2, the green curves in panels (a) and (b) correspond to the interface contribution $\beta_{\text {int }, 0}$ to the electron spin splitting, whereas the blue curves refer to the contribution $\beta_{\text {int }, 1}$. In the caption for Fig. 2 in the original variant of the paper the blue and the green curves are erroneously reversed, whereas the description of the red curve is correct. 